Remarque. - 11 y a lieu de remarquer que le terme $y$ contenant $V$ à la puissance $(-0,25)$, l'équation (2) n'est plus rigoureusement celle d'une ligne droite, et que l'équation (I) n'est plus aussi simple qu'elle en a l'air de prime abord; toutefois, $y$ varie assez peu avec $V$, car l'on a :

$$
\frac{\Delta r}{y}=-\frac{1}{4} \frac{\Delta V}{V}
$$

Si l'on a à calculer la vitesse $V$, il faut d'abord attribuer à $V$ la valeur que l'on suppose la plus approchée, pour connaitre le coefficient $y$.

A titre de comparaison, nous croyons intéressant de rappeler la formule de KutTer, qui est très employée en Amérique, et la nouvelle formule proposée en 1897 par M. Bazin (Annales des Ponts et Chaussées, tome IV).

$1^{\text {o Formule Kuller : }}$

$$
V=\frac{A+\frac{b}{n}}{\mathrm{I}+A \frac{n}{\sqrt{R}}} V \overline{R I}
$$

Dans cette formule, $n$ est un coefficient de rugosité qui tient compte de la nature des parois.

En unités américaines (pieds), il faut prendre:

$$
A=4 \mathrm{I}, 60^{\circ}+\frac{0,0028 \mathrm{r}}{\mathrm{I}} \text { et } b=\mathrm{I}, 8 \mathrm{II}
$$

En unités métriques, ces coefficients doivent être multipliés par $\sqrt{0,305}$, ce qui donne:

$$
A^{\prime}=23+\frac{0,00155}{\mathrm{I}} \text { et } \quad b^{\prime}=\mathrm{I}
$$

$2^{\circ}$ Formule Bazin:

$$
V=\frac{87}{1+\frac{\gamma}{V \bar{R}}} V \overline{R I}
$$

Y étant le coefficient de rugosité.

\section{Usine hydro-électrique de la Kern River}

Dans le courant de l'année dernière, la E'dison E'lectric $C^{\circ}$, de Los Angeles (Californie), a terminé l'aménagement d'une chute de la Kern River, pour alimenter en énergie électrique Los Angeles et les autres villes voisines de cette partie de la côte du Pacifique. La Kern River prend sa source aux glaciers des Monts Whitney (4000 m.), à l'extrémité sud de la Sierra Nevada, et est l'un des principaux affluents du San Joaquin River. Dans cet article, nous nous proposons de décrire, d'après l'Éngincering Record, les parties principales de cette installation qui comprend : un barrage de dérivation, une série de tunnels d'une longueur totale de 14 kilomètres, une conduite forcée en galerie, une usine génératrice avec unités de $10000 \mathrm{HP}$, et une ligne de transmission de 188 kilomètres, à la tension de 75000 volts.

Barrage. - Le barrage est rectiligne et submersille. Il a $62 \mathrm{~m}$. de longueur à la crète, $6 \mathrm{~m} 10$ de hauteur au-dessus du lit de la rivière, et $10^{\mathrm{m}} 66$ au-dessus du rocher de fondation. Son couronnement de $2^{\mathrm{m}} 14$ de largeur, sur lesquels $0 \mathrm{~m} 30$ seulement du côté aval est horizontal, le reste cst incliné du côté amont avec une pente de $1 / 6^{\circ}$. Le parement amont est incliné de un seizième sur toute sa hauteur. Cellai d'aval est incliné à $45^{\circ}$ sur $4^{\mathrm{m}} 57$ à partir du couronnement, puis se continue pari un are de cercle de $30^{\mathrm{m}} 48$, de telle. sorte que la largeur à la base de la fondation est de $16^{\mathrm{m}} 09$.

Un tunnel, long de $211 \mathrm{~m}$., sert à évacuer les apports dela rivière, ot au besoin pour la vidange éventuelle du réser. voir. Il scrvit tout d'abord à dériver les eaux de la Kern pendant la construction du barrage.

Le premicr tunnel débouche dans le réservoir tête gauche du balrage, et sa largeur en ce point a élé portè à $5^{\mathrm{m} / 13}$. Il est muni de vannes de garde, et d'une grillo de $6^{\mathrm{m}} \mathbf{1 0}$ de long sur $\mathbf{2}^{\mathrm{m}} \mathbf{4} 4$ de haut, composéc de Japo reaux de $76,2 \times 12,7 \mathrm{~mm}$., espacés de $76,2 \mathrm{~mm}$.

Tunnels. - Entre la prise d'eau et la chambre de miseen charge se trouvent 19 tunnels, dont la longueur varie de $152 \mathrm{~m}$. a $1330 \mathrm{~m}$.; cinc d'entre eux ont plus de $900 \mathrm{~m}$., cirin autres entre 600 et $900 \mathrm{~m}$. et quatre entre 300 et $600 \mathrm{~m}$. leur longueur totale est do $13077 \mathrm{~m}$. Ces tunnels ont the excavés suivant un rectangle, de $2^{\mathrm{m}} 743$ de large sur 2m880 de haut, surmonté d'un are de cercle de 0 '"457 de flèche, les angles de base étant remplacés par des congés de $76 \mathrm{~mm}$. Un revêtement en béton, de 152 à $244 \mathrm{~mm}$. d'épaisseur, a été appliqué contre les parois. Ce béton était dosé à raison de 1 partie de ciment pour 3 de sable et 5 de pierres cassées passant dans un anneau de $51 \mathrm{~mm}$. Sur ce béton, on a appliqué un enduit de $6,2 \mathrm{~mm}$. d'épaisseur, dosé à raison de 1 partie de ciment pour 2 de sable. Le sable et la pierre du béton étaient obtenus par broyage du granit exlrait de certains points du tunnel.

Le profil du tumnel, ainsi choisi, n'est pas le plus favoralde au point de vue de la perte de charge (car, pour cela, il aurait fallu adopter une largeur sensiblement double de la hauteur', mais c'est celui qui a paru le plus pratique a cause de l'état relativement peu solide du rocher dans cel. taines parties de la dérivation. C'est ainsi que, sur' environ 15 pour 100 de la longueur, on a dù renforcer l'épaisseur du béton à la clé de voute, et porter cette épaisseura á $30 \mathrm{cms}$, et même à $45 \mathrm{cms}$ en certains endroits.

Les caractéristiques de la partie mouillée du canal de

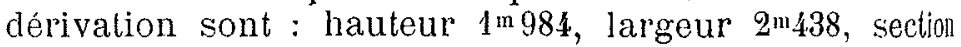
$4^{m 2} 837$, périmètre mouillé $6^{\mathrm{m}} 406$, rayon moyen $0^{\mathrm{m}}-\mathrm{T} 51$, pente 1,5 mm. par mètre. Le débit prévu, d'après la formule de KuTTER, en prenant pour colficient de rugosité la valeur 0,012 , est de 13,15 mètres cubes (").

Pour l'excavation des tunnels, on s'est servi de perforat trices à air comprimé, à raison de 2 perforatrices par front d'altaque. L'avancement moyen étail de 1 m52 par journée de 10 heures.

Aquedrics. - Afin d'aller autant que possible en ligne droite, et par cela même de réduire au minimum la lon. gueur de la dérivation, on fut amené à traver'ser des ravins au moyen do ponts-canaux, au nombro de 6, qui sont cunstruits en loois, à l'cxception du troisième qui est en béton armé. Le plus long de ces aqueducs a $314 \mathrm{~m}$., les autros varient de 15 a $51 \mathrm{~m}$. Les parois sont constituées par des planches de séquoia, dont les extrómités sont taillées en biseau de manière à présenter' à l'intérieur un joint de $6,3 \mathrm{~mm}$. qui est jouché avec de l'étoupe de bateaul. Les joints sont recouverts d'asphalte, sur laquelle est placé un léger voligeage. Sur les côtés, la moilié supérieure de ce voligeage est taillé en courbe, de manière à former un creux dans lequel on coule l'asphalte.

Le canal qui relie les tunnels no 6 et 7 (numérotés dans le sens du courant) est en béton armé. L'armature mítal. lique du canal proprement dit est constiluée par du métal déployé reposant sur des poutrelles transversales à I en

() L'application des formules précédentes donne $Y=2 m 715$. 
aciel, espacées de $1 \mathrm{~m} 22$, et fixées elles mèmes à des poutres longitudinales à I qui s'appient sur des pilier's en béton. Toute cette armature ost enrobée dans du béton ayant une épaisseur de 10,16 cms.

Il oût été désirable de pouvoir installer un résorvoir régulateur d'une assez grande capacité au départ de la la conduite forcée, malheureusement, la pente de la montagne étant voisine de $45^{\circ}$ en cel endroit, on dut se contenter do constituer une chambre n'ayant en plan qu'une section de $12 m 00-1-9 m 15$. Un déversoir, composé de 5 ouvertures de amp de longueur, et dont on peut faire varier la crète do finl, au moyen de hausses, sert à évacuer aulomatiquement los eaux de trop plein. Deux vannes de chasse sont on outre disposées au fond de la chambre. La conduite forcce part du fond de la chambre, et en avant d'elle se trouvent des grilles et des vannes d'arrêt.
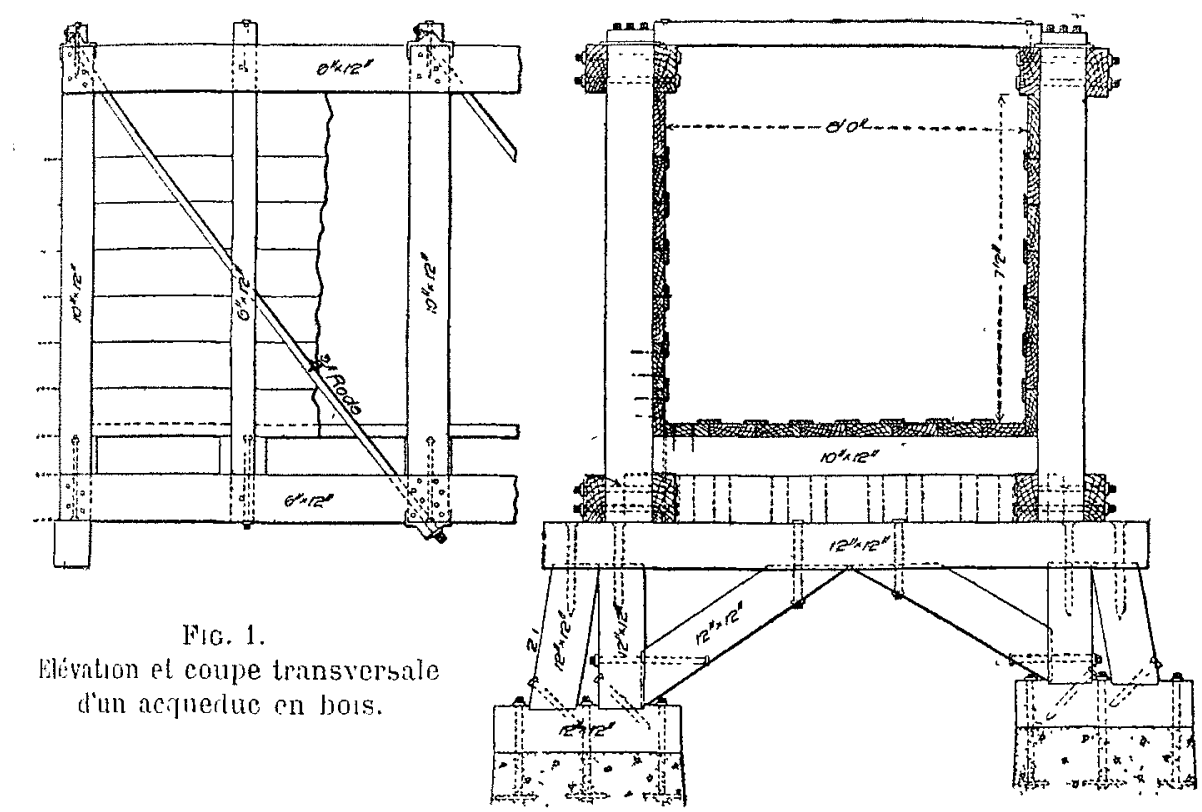

Fig. 1.

Fieviation el coupe transicrsale d'un acquerluc en bous.

Conduite forcée. - Le dispositif employé pour la conduile forcée rappelle celui qui a été employé à l'usine de Livet par la Société électroclimique de la Romanche. Cette conduite forcée est constituée par un tuyau d'acier, de 2 286 de diamétre intérieur, qui est installé dans une galeric de 2 m743 da diamètre, creusce dans lo flanc de la montagne. Lintervalle compris entre la conduite métallique et le rocher étant bourré de béton, dosć à raison de 1 de ciment pour 3 de sable et 3 de pierres cassées. Cette galerie, longue de $517 \mathrm{~m}$., a d'abord une inclinaison de 130,32 pour 100 sur $79 \mathrm{~m}$. de longueur, puis de 101,35 pour 100 sur $10 \mathrm{~m}$., et enfin de 84,93 pour 100 sur $303 \mathrm{~m}$. Sur lo reste de la longueur, soit $125 \mathrm{~m}$., elle est horizontale. A son entrée dans la chambre de mise en charge, la conduite est tronconique sur $6 \mathrm{~m} 10$ de longueur, avec un diamètre d'ouverture de 3 m05. Sur les 74 derniers metres do la partie hnizontale, la conduite traverse des éboulis. Le diamètre de la conduite a alors été réduit à $1 \mathrm{mb0}$. Aucun béton n'a été cmployé autour de cette conduite, on a seulement laissé en place les boisages qui étampaient la galerie.

La conduite ful montée par troncons de 3 mos en commençant pap le has, chaque troncon était hissé par un cable aérien jusquà la chambre de mise en charge, puis, te lá, les troncons étaient redescendus dans la galerie au moyen de chariots se déplaçant sur deux rails formant une pelite vole de service a l'interieur de cette galeric. A cet effet, deux poutres étaient placées à l'intérieur du tuyau, et venaient s'appuyer sur un chariot à l'avant et à l'arrière.

Une fois le montage de la conduite terminée, on procéda àson inspection, et l'on constata en certains endroits, en tapant avec un martcau sur la paroi intérieure du tuyau, qu'il s'était produit à la partie inférieure des poches dans le béton, cos poches provenant de la difleulté quiil y avait à hien pilonner le béton dans les creux du rocher par suite de l'irrégularité de lexcavalion. Pour boucher ces poches, on perça des trous dans la tóle do la conduite, ot l'on fil des injections de morticr; puis, les trous furont obturós au moven de tampons. On fit ainsi 116 injectiens.

Usine génératrice. - L'usine génératrice est construite à l'altitude de $324 \mathrm{~m}$. au-dessus du niveau de la mer, immédiatement en avant do la prise d'eau de l'usine hydro-

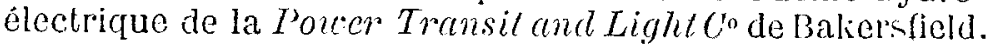
La puissance moyenne de l'usine est de $20000 \mathrm{kw}$, et sa puissance maxima de 30000 kilowatts.

L'usine comporte 4 puissants groupes électrogènes, de $107 \check{0} \mathrm{HP}$. chacun, composís d'un alternateul actionné à chacune de ses extrémités par uno roue Pelton, et de 2 groupes d'excitation.

Les roues Pelton des alternateurs travaillent normalement sous une charge d'eau de $201 \mathrm{~m}$, et ont une vitesse de rotation de 250 tomrs par minute. Elles sont constituees par un disque

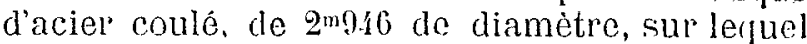
sont montés 18 augrets en bronze, du type ordinaire, ayant $698,5 \mathrm{~mm}$. de largeur. La huclio est en Ponte. Le moment d'incrtic polaire de la partie tournante d'un groupe olectrogèno est de $76000 \mathrm{kgm}^{2}$. D’après les spécifications du contrat la variation de vitesse ne devait pas excéder 7,$8 ; 3,5$ et 1,7 pour 100 , pour des rariations subites de la charge de 100; 50 et 25 pour 100 . Le rendement garanti devait, en outre, atteintre 82,5 pour 100 . Ces roues proviennent des atcliers de l'Allis Chalmers Company.

Les excitatrices sont actionnées chacune par une roue Pelton montée a l'une de leul's extrémités. Du côté opposé à la roue se trouve un volant.

La régulation de la vitesse des rouos se fait de deux manières distinctes: $t^{\circ}$ une aiguille centrale, mancruvrée à la main, augmente ou dıminue la section du passage de l'cau; 20 un régulateur automatique, à servo moteur à huile sous pression, agil sur la buse do distribution pour dévier plus ou moins le jet et, par cela même, aurmenter ou diminuer la puissance de la loue. Ce dispositif a lavantage de maintenir une vitesse constante dans la conduite forcée, et, par suite, d'éviter linfluence néfaste des coups de bélier $\left(^{\star}\right)$.

Ia huse de distribution de chaque roue est constiluéc par deux tuyaux en forme d' $\langle>$, I'Y d'amont étant fixe ct horizontal, celui d'aval étant mobilo verticalement autour d'un axe horizontal passant par les extrémités des branches inclinées. Le poids de la partie mobile de la buso est en partie équilibré par un piston supportant sur une de ces faces la pression de l'eau. Il en est de même pour la prossion de l'eau qui agit sur l'aiguille de réglage des buses.

Chaque groupe générateur, alternatcur ou excitalrice, est relié par une chaîne à un régulateur Lombard. Celui-ci est equipé de telle façon que l'on puisse faire à volonté l'une des quatre opérations suivantes : 1" Régulation purement manuelle; $2^{\circ}$ régulation automatique ordinaire; 30 réculation à la main, tout en se servaut du servo-moteur a hiuile, les boules du tachymôtre étant débrayéos; fo rógulation faite du tableau, de distribution, au moyen d'un potit moteur électrique. En général, les aiguilles sont maintonues à une position fixe déterminée, ot le ruglage instantané esl fait par le régulateur.

(*) Un disposilif analogue a dbe employé í l'usine liydro-dlectrugue de Plke's Peak (Vorr La Houile Blanche de novembre 1906). 
En marche normale, les aiguilles sont ouvertes en plein, le jet a, alors, a la sortic des busos, un diamètre de $187 \mathrm{~mm}$. et une vilesse de $72 \mathrm{~m}$. a la seconde. Lorsqu'il est dévié, ce jet vient frapper obliquement une cuirasse courbe on acier, qui se termine par une paroi verticale le long de lacpuclle l'eau tombe dans le canal de fuite.

Les alter nateur's produisent du courant tripliasé a 2300 volts et 50 periodes. Leurs axes sont suppoptes par deux paliers entro lesquels sont calés les inducteurs mobiles, les roues Pelton étant montcies en porte à faux. Les coussinets portent, à leur partie inférieure, un certain nombre de petils trous racevant de l'luuile à la pression de $70 \mathrm{krgs}$ par $\mathrm{cm}^{2}$, de manière a controbalancer lo poids de la partie mobile sur les coussinets.

Les excitatrices ont une puissance de $225 \mathrm{kr}$, et produisent du courant continu ì la tension de 125 volts, à la vitcsse de 430 tours par minute.

La station comprend 13 transformateurs monophasés, de 1167 livs, a bain d'huile, qui forment 4 groupes de 3 apparcils. Il y a, en outre, un transformateur supplémentaire de réserve. Ils sont connectés en triangle au primaire, ou ils recoivent le courant des alternateurs à 23000 volts entre fils, of sont connectés en étoile au secondaire, ouils élèvent le courant de Jigno à 75000 volts. Des bornes ont été ménagées pour pouvoir recueillir du courant à 56250 et 37500 volts.L'huile est envoyéo aux transformateurs sous une très légère pression, et elle retourne par gravité aux réfrigérants.

Le tableau général do. mine la salle des machines, et est divisé en 9 panneaux : 1 pour les services auxiliaires, 2 pour les excitatrices, 4 pour les alternatcurs, 1 pour les Peeder's; le der'nier est vide pour le moment. Les coupe-circuits à 75000 volts sont logés à raison de 1 par phase dans des cellules séparées en ciment.

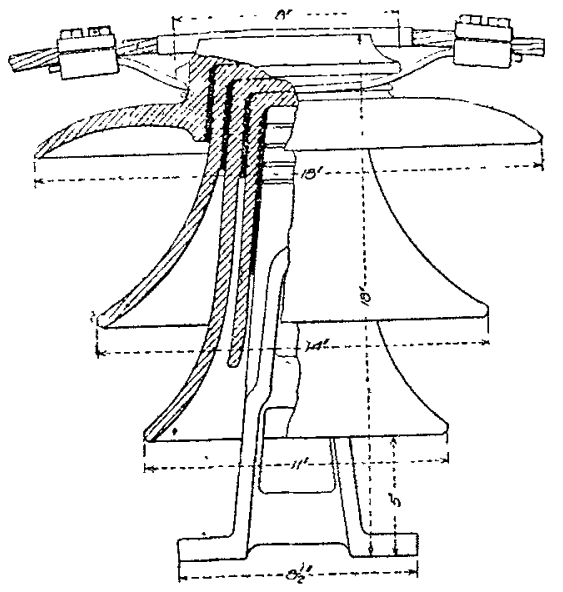

Fig. 2. - Isolaleur à 60000 rolls.

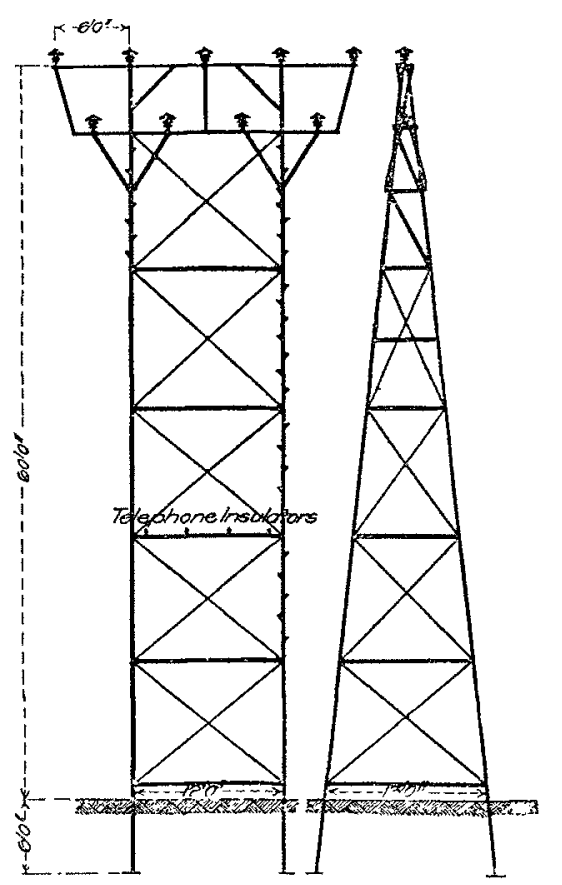

Fig. 3. - Poteau mélallique.
Ligne de transmission. - La ligne de transport d'énergie, qui reliel'usine génératrice à la sous-station de Los Angeles, à $188 \mathrm{kms}$ de longueur. Elle est supportée par des pylônes métalliques, ayant de 9 à $18 \mathrm{~m}$. de hauteur, espacés de $210 \mathrm{~m}$. Entre chacun de ces pylônes, se trouvent 2 poteaux en bois, mais les pylônes et les fils sont capables de travailler sous la plus grande portée. Le voltage n'est actuel lement que de 60000 volts, mass il sera bientót porté à la tension de 75000 volts.

La ligne comporte 3 circuits distincts, les fils étant espacés de 1 m829. Le cuivre employé a une résistance de rupture de $44 \mathrm{kgs}$ par $\mathrm{mm}^{2}$. Les isolateurs ont $437 \mathrm{rnm}$. comme hauteur et comme largeur maxima; ils sont l'at'leurs représentés par la figure 2. Les spócifications de la fourniture stipulaient qu'ls doivent pouvoir resister a un tension de 100000 volts sous une pluie faisant un angle do $30^{\circ}$ avec la verticale, et correspondant à uno hauteur d'au tombée de $25,4 \mathrm{~mm}$. en 5 minutes. A sec, la rósistance doil être de 150000 volts pendant 30 sccondes.

Remarques. - Les promières études ont commencó cll 1901, et los travaux débutèrent à la fin de 19012, puis so poursuivirent jusqu'en mai 1907, ćpoque à laquolle linstal. lation a été complètement terminéc. La Compagnie Ldison ayant installé deux autres usines en 1904, les travaur furent peu activés pendant cette année là. L.e 11 mai 1907, leau fut mise dans los tunnels, ot, le 19 maj, 2.50) lins étaient transmis a Los Angeles.

Pendant les travaux, une petite usine de 400 livs fut installée, utilisant une chute de $12 \mathrm{~m}$., au moyed do 2 turbines Mac Cormick à réaction, qui actionnaient chacumom. alternateur à 2300 volts. Lirnergie était transmisc aux divers chantiers à la tension de 10000 volts.

La station réceptrice de Los Angeles contient actuelle. ment 3 groupes électrogènes turbo-alternateurs, du systemo Curtis, de la Général Electric $C^{\circ}$, de 2000 et 2300 livs, rui ont été installés en 1903, et un autre horizontal systeme Westinghouse-Parsons de $6000 \mathrm{kws}$, installé à la fin de 1500.

La Edison Electric $C^{\circ}$ de Los Angeles possède encore 6 autres usines, produisant ensemble $8700 \mathrm{krvs}$ et ótaljlies sur la Santa Anna River et sur les Mill Creek et Lytle Crcok. Elle a, en outre, acquis les droits nécessaires pour l'instal. lation de 4 autres usines sur le cours supérieur de la Kern River, capables de produire $46600 \mathrm{kws}$. Elle s'est auss intéressée pour deux tier's dans les droits de riveraineté de la King's River, ou l'on pourra produire 95000 kivs. I Compagnie alimente actuellement, en énergie ólectrique, 16 villes ayant au total 420000 habitants. Son capital cst de 11200000 dollars, dont 4000000 sous forme d'actions privilégiées.

J. MATuEU.

\section{THÉORIE DU DÉVERSOIR}

\section{Notes de M. Boussineso, de l'Académie des Sciences (Suite) \\ Sur la théorie des déversoirs épais, ayant leur seuil horizontal et évasé ou non à son entrée ( $\left.{ }^{\star}\right)$.}

VI. - Concevons maintenant que la face inférieure de la nappe déversante, au lieu de subir une pression constante donnée, puisse être astreinte, par une conformation appropriée du seuil non plus étroit, mais assez large, à prendic près de la section contractée une certaine courbure, indépendante des pressions qu'elle supportera. Imaginons, par exemple, que, le seuil étant plan et sensiblement horizontal, on parvienne, au moyen d'un évasement convenable de son entrée, à y supprimer les cspaces morts qu'occupe une call dormante ou tourbillonnante, c'est-à-dire dont le rayon $R_{*}$ de courbure, sur la section contractée, sera dès lors infini, comme l'admettait Bélanger. La troisième formule (15) montre que cette hypothèse revient à poser $k=I$, et que, plus généralement, la supposition d'un rayon $R_{0}$ de courbure en rapport donné constant avec l'épaisseur $\eta$ équivaut à fixer pour $k$ une certaine valeur connue. Dès lors, l'expres.

(") Séance du 17 octobre 1887 . 